Pacific Journal of Mathematics

ON THE LU QI-KENG CONJECTURE AND THE BERGMAN 


\title{
ON THE LU QI-KENG CONJECTURE AND THE BERGMAN REPRESENTATIVE DOMAINS
}

\author{
Shozo MATSUURA
}

The main purpose of this paper is to give the affirmative solution for the Lu Qi-Keng conjecture in the case of bounded complete circular domains.

Some results are that Bell's proposition, which is related to the evaluation about the Bergman kernel functions of homogeneous complete circular domains, is extended to the case of bounded complete circular domains and that the Bergman representative functions with respect to $z_{0}(\neq 0)$ of any bounded and any homogeneous bounded complete circular domain with center at the origin are biholomorphic and isomorphic (biholomorphic in the narrow sense), respectively.

1. Introduction. The Bergman kernel function $k_{D}(z, \bar{t})$ of a domain $D$ in $C^{n}$ plays important roles in the theory of biholomorphic mappings because of its biholomorphic relative invariance (see (15)).

Lu Qi-Keng [10] introduced the conjecture, which is still open, that "The equation $k_{D}(z, \bar{t})=0$ has no solution in $D \times D^{*}$, if $D$ in $C^{n}$ is a bounded simply connected schlicht domain", where $D^{*}=$ $\{\bar{t} \mid t \in D\}$ and the symbol denotes the conjugate.

It is known that in general multiply connected schlicht domains do not satisfy the Lu Qi-Keng conjecture ([11] Theorems 1, 3; [12] Example). But in the case of simply connected schlicht domains in $C^{n}(n \geqq 2)$ it seems that we have no example which does not satisfy the conjecture. In $C$ it is easily seen by the Riemann's mapping theorem that any simply connected schlicht domain satisfies the conjecture.

In $\S 2$ we shall show that any bounded complete circular domain satisfies the Lu Qi-Keng conjecture, and by using this result extend the following proposition of Bell [1], which is recently proved:

Proposition. Let $D$ be any homogeneous domain in $C^{n}$, n-dimensional complex space, such that if $z \in D$ and $\lambda \in C$ and $|\lambda| \leqq 1$ then $\lambda z \in D$ (i.e., $D$ is a complete circular domain with center at the origin). Then given a compact subset $H$ of $D$ there are constants $a_{H}>0$ and $b_{H}<\infty$ such that for all $z \in D$ and $t \in H$

$$
a_{H} \leqq\left|k_{D}(z, \bar{t})\right| \leqq b_{H}
$$

where $k_{D}$ denotes the Bergman kernel function of the domain $D$.

Throughout this paper we shall call a holomorphic mapping $w=$ 
$f(z) \equiv{ }^{t}\left(f_{1}(z), \cdots, f_{n}(z)\right), z \equiv{ }^{t}\left(z_{1}, \cdots, z_{n}\right) \quad\left({ }^{t}():\right.$ transpose $)$, of a schlicht domain in $C^{n}$ (i) a biholomorphism if $f(z)$ is holomorphic and the Jacobian $J_{f}(z) \neq 0$ in $D$, i.e., both $f$ and the inverse $f^{-1}$ are holomorphic and $f$ is locally one-to-one (one-to-one in the sense of Riemann regions (see [3], p. 117)), (ii) an isomorphism if $f(z)$ is biholomorphic and globally one-to-one (both $D$ and $f(D)$ are schlicht domain in $C^{n}$ ), and (iii) an automorphism if $f(z)$ is an isomorphism of $D$ onto itself, respectively.

In $\S 3$ we treat the representative domain introduced by $\mathrm{S}$. Bergman [2] as a canonical domain of the biholomorphic equivalent class of a bounded schlicht domain in $C^{n}$ corresponding to the unit disc in $C$ in the Riemann's mapping theorem. The function which maps a domain $D$ onto the representative domain of the biholomorphic equivalent class of $D$ is called the representative function of $D$ (see (10)). In general the representative function $w=w(z)$ of $D$ with respect to a fixed point $z_{0} \in D$ is singular at any point $z \in\left\{z \in D \mid k_{D}\left(z, \bar{z}_{0}\right)=0\right\}$ and further is not locally one-to-one at any point $z \in\{z \in D \mid$ Jacobian $\left.J_{w}(z)=0\right\}$. In these cases the representative domain does not belong to the biholomorphic equivalent class of $D$, and this weaken the representativity of the representative domain of the biholomorphic equivalent class of $D$.

We shall prove that the representative function of any bounded complete circular domain $D$ with respect to $z_{0} \in D$ is biholomorphic, and that in the case of homogeneous bounded complete circular domains the representative function is an isomorphism. We give an example in the case of Cartan domains. Finally, we treat the two sorts of $m$-representative domains of Maschler [7] and Kato [4] or Kikuchi [5] and give an example in the case of a unit disc.

The author wishes to express his gratitude to Professor S. Ozaki for helpful discussions to this paper.

\section{Lu Qi-Keng conjecture.}

Definition. A domain $D \subset C^{n}$ is called a $\mathrm{Lu}$ Qi-Keng domain if the equation

$$
k_{D}(z, \bar{t})=0
$$

has no solution in $D \times D^{*}$ ([10], [11], [12]).

If (1) has no solution in $D \times E^{*}$, where $E^{*}=\{\bar{t} \mid t \in E \subset D\}$, it is said that $k_{D}(z, \bar{t})$ or $D$ satisfies the Lu Qi-Keng conjecture for a subset $E^{*}$ of $D^{*}$.

Lemma 1. Assume that the Bergman kernel function $k_{D}(z, \bar{t})$ of a bounded domain in $C^{n}$ satisfies the Lu Qi-keng conjecture at a 
point $\bar{t}_{0} \in D^{*}$. - Let us set

$$
\Omega_{\Delta}=\left\{t \in D \mid k_{D}(z, \bar{t}) \neq 0 \text { for } z \in \Delta\right\},
$$

where $\Delta$ is an arbitrary compact subset of $D$. Then $\Omega_{\Delta}$ must be an open set in $D$.

Proof. Let us take an arbitrary point $\tau \in \Omega_{\Delta}(\neq \varnothing)$. Then it follows that $k_{D}(z, \bar{\tau}) \neq 0$ for $z \in \Delta$. As it is well known that $k_{D}(z, \bar{t})$ is holomorphic with respect to $2 n$ complex variables $(z, \bar{t}) \in D \times D^{*}$, there exists a neighborhood $U\left(z_{\alpha}, \bar{\tau}\right)=U\left(z_{\alpha}\right) \times U_{z_{\alpha}}(\bar{\tau})$ for each point $z_{\alpha} \in \Delta$ such that $k_{D}(z, \bar{t}) \neq 0$ for $(z, \bar{t}) \in U\left(z_{\alpha}, \bar{\tau}\right)$, where $U\left(z_{\alpha}\right)$ and $U_{z_{\alpha}}(\bar{\tau})$ are neighborhoods of $z_{\alpha}$ and $\bar{\tau}$, respectively. Since $\bigcup_{\alpha} U\left(z_{\alpha}\right)\left(z_{\alpha} \in \Delta\right)$ is an open covering of the compact set $\Delta, \Delta$ has a finite covering $\bigcup_{i=1}^{m} U\left(z_{i}\right) \supset \Delta$, where $z_{i} \in \Delta \quad(i=1,2, \cdots, m)$. If we set $U(\bar{\tau})=$ $\bigcap_{i=1}^{m} U_{z_{i}}(\bar{\tau}), U(\bar{\tau})$ is an open neighborhood of $\bar{\tau}$ and $k_{D}(z, \bar{t}) \neq 0$ in $\Delta \times U(\bar{\tau})$. This shows that $\Omega_{\Delta}$ is an open set in $D$.

REMARK 1. It is clear that $k_{D}(z, \bar{t}) \neq 0$ in $\bigcup_{i=1}^{m} U\left(z_{i}\right) \times U(\bar{\tau}) \supset$ $\Delta \times U(\bar{\tau})$.

REMARK 2. A bounded complete circular domain $D$ with center at $t_{0}$ is a Bergman minimal domain with the same center (see [6]). Therefore, $k_{D}(z, \bar{t})$ satisfies the Lu Qi-Keng conjecture at $\bar{t}=\bar{t}_{0}$, for a domain $D$ is a Bergman minimal domain with center at $t_{0}$ if and only if

$$
k_{D}\left(z, \bar{t}_{0}\right)=k_{D}\left(t_{0}, \bar{t}_{0}\right)>0 \text { for } z \in D([6]) \text {. }
$$

Lemma 2. Let $D$ be a bounded complete circular domain with center at the origin, then $\Omega_{\Delta}=D$, that is, $k_{D}(z, \bar{t}) \neq 0$ for $(z, \bar{t}) \in \Delta \times$ $D^{*}$, where $\Delta$ is an arbitrary compact subset of $D$.

Proof. It is easily seen that $D$ is starlike with respect to the origin (see Fuks [3], p. 45). If $\Omega_{\Delta} \neq D$, there exists a point $\omega \in D$ such that $k_{D}(\zeta, \bar{\omega})=0$ for some $\zeta \in \Delta$ and $k_{D}(z, \lambda \bar{\omega}) \neq 0$ for all $z \in \Delta$ and $\lambda \in[0,1)$, because by Lemma $1 \Omega_{\Delta}$ is an open set including the origin and $D$ is starlike. $\omega$ lies in $\partial \Omega_{\Delta} \cap D$ and does not lie in $\Omega_{\Delta}$.

In the case of circular domains with center at the origin, by the Cartan's theorem, the Bergman kernel function $k_{D}(z, \bar{t})$ of $D$ has the form

$$
k_{D}(z, \bar{t})=\sum_{k=0}^{\infty} \overline{\Phi_{k}(t)} \Phi_{k}(z)=\sum_{k=0}^{\infty} \overline{A_{k} t^{k}} A_{k} z^{k},
$$

where $z^{k}=z \times \cdots \times z$, the symbol $\times$ denotes the Kronecker product 
here and after, and $A_{k}$ is a $1 \times n^{k}$ constant coefficient matrix and $\left\{\Phi_{0}, \Phi_{1}(z), \Phi_{2}(z), \cdots\right\}\left(\Phi_{k}(z)=A_{k} z^{k}(k \geqq 1)\right.$ : homogeneous polynomial of degree $k$ and $\Phi_{0}=A_{0}$ : nonzero constant) is a complete orthonormal system of the Hilbert space of square integrable holomorphic functions on $D$.

As $\omega \in \partial \Omega_{\Delta} \cap D$ and $\omega \notin \Omega_{\Delta}$, we have $\lambda \bar{\omega} \in \Omega_{\Delta}^{*}=\left\{\bar{t} \mid t \in \Omega_{\Delta}\right\}$ for all $\lambda \in[0,1)$. Let us consider a point $\lambda \bar{\omega}$ in place of $\bar{\tau}$ in the proof of Lemma 1 and $\bigcup_{i=1}^{m(\lambda)} U_{\lambda}\left(z_{i}\right)$ to be an open covering of $\Delta$ corresponding to $\lambda \bar{\omega}$. As we may take $1-\lambda(0<\lambda<1)$ as small as we need, there exists $\lambda_{0} \in(0,1)$ such that $\lambda_{0} \omega \in \Omega_{\Delta}$ and $\zeta / \lambda_{0} \in U_{\lambda_{0}}\left(z_{i}\right)$ for some $i$, because $\zeta$ (a critical point in $\Delta$ for $\omega$ ) belongs to $U_{\lambda_{0}}\left(z_{i}\right) \cap \Delta$ for some $i$ and $U_{\lambda_{0}}\left(z_{i}\right)$ is open. By Remark 1, for this $\lambda_{0} k_{D}\left(\zeta / \lambda_{0}, \lambda_{0} \bar{\omega}\right) \neq 0$ holds. On the other hand, using the idea of D. Bell [1], we have by (3)

$$
k_{D}\left(\zeta / \lambda_{0}, \lambda_{0} \bar{\omega}\right)=\sum_{k=0}^{\infty} \lambda_{0}^{k} \overline{A_{k} \omega^{k}}\left(1 / \lambda_{0}^{k}\right) A_{k} \zeta^{k}=k_{D}(\zeta, \bar{\omega})=0,
$$

which is contradictory. This completes the proof.

THEOREM 1. Any bounded complete circular domain with center at the origin is a Lu Qi-Keng domain.

Proof. By Lemma $2, k_{D}(z, \bar{t}) \neq 0$ for $(z, \bar{t}) \in \Delta \times D^{*}$. Thus we obtain $k_{D}(z, \bar{t}) \neq 0$ for $(z, \bar{t}) \in D \times D^{*}$, since $\Delta$ is an arbitrary compact subset of $D$.

REMARK 3. Theorem 1 holds for any bounded complete Reinhardt circular domain, because Reinhardt circular domains are (Carathéodory) circular domains.

A homogeneous domain $D$ for which $k_{D}(z, \bar{t})$ satisfies the Lu Qi-Keng conjecture at a point $\bar{t}_{0} \in D^{*}$ is a Lu Oi-Keng domain by the homogeneity of $D$ and the biholomorphic relative invariance (see (15)) of the Bergman kernel function (c.f. [1]). Therefore, any homogeneous complete circular domain $D$ is a Lu Qi-Keng domain, because $D$ satisfies the Lu Qi-Keng conjecture at the center by Remark 2.

Theorem 1 leads to the following extension of the Bell's proposition.

Corollary 1. The Bell's proposition valid for any bounded complete circular domain $D$ in $C^{n}$.

Proof. In the proof of the Bell's proposition it is essential that a domain $D$ is a Lu Qi-Keng domain and a complete circular one. From Theorem $1 D$ is a Lu Qi-Keng domain. Therefore, by using the same procedure of the proof of the Bell's proposition, for any 
bounded complete circular domain the result is obtained.

3. Biholomorphicity of representative functions. For convenience, first we note some notations and differential formulas. $I_{k}$ shows the identity matrix of order $k$ ( $k$ : positive integer), and the symbol * denotes the transposed conjugate. $\partial / \partial z$ and $\partial / \partial z^{*}$ denote the differential operators:

$$
\partial / \partial z \equiv\left(\partial / \partial z_{1}, \cdots, \partial / \partial z_{n}\right) \text { and } \partial / \partial z^{*} \equiv{ }^{t}\left(\partial / \partial \bar{z}_{1}, \cdots, \partial / \partial \bar{z}_{n}\right) .
$$

Let holomorphic functions $A, B$, and $b$ of several complex variables $z \equiv{ }^{t}\left(z_{1}, \cdots, z_{n}\right)$ and $\bar{z} \equiv{ }^{t}\left(\bar{z}_{1}, \cdots, \bar{z}_{n}\right)$ be $(k \times l),(l \times m)$ and $(n \times 1)$ matrices, respectively. Then the following differential formulas can be ascertained:

$$
\partial(A B) / \partial z \equiv(\partial / \partial z) \times(A B)=(\partial A / \partial z)\left(I_{n} \times B\right)+A(\partial B / \partial z),
$$

$$
\partial(A \times b) / \partial z \equiv(\partial / \partial z) \times(A \times b)=(\partial A / \partial z) \times b+A \times(\partial b / \partial z)
$$

and

( 8 ) $\partial A / \partial z \equiv(\partial / \partial z) \times A=(\partial A / \partial w)\left((d w / d z) \times I_{l}\right), \quad d w=(d w / d z) d z$, where $A=A(w, \bar{w})$ and $w=w(z) \equiv{ }^{t}\left(w_{1}(z), \cdots, w_{n}(z)\right)$ is a holomorphic vector function of $z$ and $d z \equiv{ }^{t}\left(d z_{1}, \cdots, d z_{n}\right)$ (see [4], [5], [9]).

If there exists a primitive function $F(z)$ of a function $A(z)$ such that $d F(z) / d z=A(z), \int_{z_{0}}^{z} A(z) d z=F(z)-F\left(z_{0}\right)$ is called the integral of $A(z)$ from $z_{0}$ to $z$, where pathes of integral are homotopic in the domain.

It is known that the function $w(z)$ which maps a domain $D$ onto the representative domain $D_{w}$ of the biholomorphic equivalent class of $D$ under the initial conditions

$$
w\left(z_{0}\right)=\tau \text { and } d w\left(z_{0}\right) / d z=I_{n}, \quad z_{0} \in D,
$$

is given in the matrix representation by making use of the Bergman kernel function as follows:

$$
w(z)=T_{D}^{-1}\left(z_{0}, \bar{z}_{0}\right) \int_{z_{0}}^{z} T_{D}\left(z, \bar{z}_{0}\right) d z+\tau,
$$

where $w(z) \equiv{ }^{t}\left(w_{1}(z), \cdots, w_{n}(z)\right), z \equiv{ }^{t}\left(z_{1}, \cdots, z_{n}\right) \in D$ and

$$
T_{D}(z, \bar{t}) \equiv \partial^{2} \log k_{D}(z, \bar{t}) / \partial t^{*} \partial z \quad \text { (see [9], [13]) . }
$$

We call $\tau$ the center of the representative domain of $D . T_{D}(z, \bar{z})$ is the Bergman metric tensor and positive definite and $T_{D}(z, \bar{t})$ is holomorphic with respect to $(z, \bar{t}) \in D \times D^{*}$ if $D$ is a Lu Qi-Keng domain. If $D$ is a bounded complete circular domain with center at the 
origin, $D$ itself is the representative domain with the same center ([6]). It is known that a domain $D$ is the representative domain with center at $t_{0} \in D$ if and only if

$$
T_{D}\left(z, \bar{t}_{0}\right)=T_{D}\left(t_{0}, \bar{t}_{0}\right) \text { for } z \in D \text { ([9], [13]) . }
$$

Hence for a bounded complete circular domain $D$ with center at the origin $\operatorname{det} T_{D}(z, 0)=\operatorname{det} T_{D}(0,0) \neq 0($ precisely $>0)$ for $z \in D$.

Lemma 3. Let $D$ be a bounded complete circular domain with center at the origin, then

$$
\Lambda=\left\{t \in D \mid \operatorname{det} T_{D}(z, \bar{t}) \neq 0 \text { for } z \in \Delta\right\},
$$

where $\Delta$ is an arbitrary compact subset of $D$, is an open set.

Proof. From (11), by calculations we have

$$
T_{D}(z, \bar{t})=\left[k_{D}(z, \bar{t}) \partial^{2} k_{D}(z, \bar{t}) / \partial t^{*} \partial z-\partial k_{D}(z, \bar{t}) / \partial t^{*} \partial k_{D}(z, \bar{t}) / \partial z\right] / k_{D}^{2}(z, \bar{t}) .
$$

(13) shows that $\operatorname{det} T_{D}(z, \bar{t})$ is holomorphic with respect to $(z, \bar{t}) \in D \times$ $D^{*}$, because by Theorem $1 D$ is a Lu Qi-Keng demain, namely, $k_{D}(z, \bar{t}) \neq 0$ in $D \times D^{*}$. On the other hand, $\operatorname{det} T_{D}(z, 0)=\operatorname{det} T_{D}(0,0)>0$ for $z \in D$. Thus, by a proof similar to the proof of Lemma 1, we have the result.

Lemma 4. Let $D$ be a bounded complete circular domain with center at the origin, then $\Lambda=D$, that is, $\operatorname{det} T_{D}(z, \bar{t}) \neq 0$ for $(z, \bar{t}) \in$ $D \times D^{*}$.

Proof. By the matrix differentiations (6) and (7) we have

$$
\partial A_{k} z^{k} / \partial z=A_{k} \partial z^{k} / \partial z=A_{k}\left(I_{n} \times z^{k-1}+z \times I_{n} \times z^{k-2}+\cdots+z^{k-1} \times I_{n}\right)
$$

and $\partial \overline{A_{k} t^{k}} / \partial t^{*}=\left(\partial A_{k} t^{k} / \partial t\right)^{*}$. Therefore, from (3) we have

$$
\begin{gathered}
\partial^{2} k_{D}(z, \bar{t}) /\left.\partial t^{*} \partial z\right|_{z=z_{1} / \lambda, t=\lambda t_{1}}=\partial^{2} k_{D}\left(z_{1} / \lambda, \lambda \bar{t}_{1}\right) / \partial t^{*} \partial z \\
=\partial^{2} k_{D}\left(z_{1}, \bar{t}_{1}\right) / \partial t^{*} \partial z, \\
\partial k_{D}(z, \bar{t}) /\left.\partial z\right|_{z=z_{1} / \lambda, t=\lambda t_{1}}=\partial k_{D}\left(z_{1} / \lambda, \lambda \bar{t}_{1}\right) / \partial z=\lambda \partial k_{D}\left(z_{1}, \bar{t}_{1}\right) / \partial z
\end{gathered}
$$

and

$$
\partial k_{D}(z, \bar{t}) /\left.\partial t^{*}\right|_{z=z_{1} / \lambda, t=\lambda t_{1}}=(1 / \lambda) \partial k_{D}\left(z_{1}, \bar{t}_{1}\right) / \partial t^{*},
$$

for the expansion (3) converges uniformly. From (13) and the above relations we arrive at

$$
T_{D}\left(z_{1} / \lambda, \lambda \bar{t}_{1}\right)=T_{D}\left(z_{1}, \bar{t}_{1}\right)
$$


for $1-\lambda(0<\lambda<1)$ as small as we need. Noting that $\Lambda$ is an open set including the origin, by the similar way as in the proof of Lemma 2 , we obtain $\Lambda=D$.

THEOREM 2. The representative function of any bounded complete circular domain with center at the origin under the initial conditions (9) is biholomorphic.

Proof. As $\Delta$ in Lemma 3 is an arbitrary compact subset of $D$, then $\operatorname{det} T_{D}(z, \bar{t}) \neq 0$ in $D \times D^{*}$ by Lemma 4 . Therefore, for the representative function (10) for an arbitrary $z_{0} \in D$ we have

$$
\operatorname{det}(d w(z) / d z)=\operatorname{det} T_{D}\left(z, \bar{z}_{0}\right) / \operatorname{det} T_{D}\left(z_{0}, \bar{z}_{0}\right) \neq 0 \text { for } z \in D,
$$

which shows that the representative function (10) under the initial conditions (9) is locally one-to-one in $D$. On the other hand, Theorem 1 shows that (10) is holomorphic in $D$. Thus the representative function is biholomorphic in $D$ with respect to an arbitrary point $z_{0} \in D$.

REMARK 4. Theorems 1 and 2 are valid for domains in the sence of Riemann regions (which become simply connected Kaehler manifolds in the present case) belonging to the biholomorphic equivalent class and in particular for domains belonging to the isomorphic equivalent class of a bounded complete circular domain by virtue of the relative invariances:

$$
k_{D}(z, \bar{t})=k_{E}(X(z), \overline{X(t)}) \overline{\operatorname{det}(d X(t) / d z)} \operatorname{det}(d X(z) / d z)
$$

and

$$
T_{D}(z, \bar{t})=(d X(t) / d z)^{*} T_{E}(X(z), \overline{X(t)})(d X(z) / d z),
$$

where $D$ is a bounded schlicht domain in $C^{n}, X(z)$ is a biholomorphism and $E$ denotes the image manifold of $D$ under $X(z)$ ([2]).

THEOREM 3. The representative function (10) of any homogeneous complete circular domain $D$ with center at the origin under the initial conditions (9) is an isomorphism.

Proof. For the representative function (10) with respect to an arbitrary point $z_{0} \in D$, let us assume $\eta=\eta(z)$ is a holomorphic homogeneous transformation (automorphism) which maps $z_{0}$ to the origin (center of $D$ ), by (16) we have

$$
T_{D}\left(z, \bar{z}_{0}\right)=\left(d \eta\left(z_{0}\right) / d z\right)^{*} T_{D}(\eta(z), 0)(d \eta(z) / d z) \text { for } z \in D,
$$


and the representative function

$$
\begin{aligned}
w(z) & =T_{D}^{-1}\left(z_{0}, \bar{z}_{0}\right) \int_{z_{0}}^{z} T_{D}\left(z, \bar{z}_{0}\right) d z+\tau \\
& =\left(d \eta\left(z_{0}\right) / d z\right)^{-1} T_{D}^{-1}(0,0) \int_{0}^{\eta} T_{D}(\eta, 0) d \eta+\tau
\end{aligned}
$$

by (16) and (8). Since $D$ itself is a representative domain with center at the origin $([6]), T_{D}(\eta, 0)=T_{D}(0,0)>0$ follows from (12). Thus we obtain

$$
w=\left(d \eta\left(z_{0}\right) / d z\right)^{-1} \int_{0}^{\eta} d \eta+\tau=\left(d \eta\left(z_{0}\right) / d z\right)^{-1} \eta+\tau .
$$

If $z_{1} \neq z_{2}, z_{1}, z_{2} \in D$, from the transitivity of $\eta(z)$ it follows that $\eta\left(z_{1}\right) \neq \eta\left(z_{2}\right)$. Let us set $w\left(z_{1}\right)=w_{1}$ and $w\left(z_{2}\right)=w_{2}$, then we have $w_{2}-w_{1}=\left(d \eta\left(z_{0}\right) / d z\right)^{-1}\left(\eta\left(z_{2}\right)-\eta\left(z_{1}\right)\right) \neq 0$, because $\operatorname{det}\left(d \eta\left(z_{0}\right) / d z\right) \neq 0$. This shows that the representative function $w=w(z)$ is globally one-to-one in $D$.

Example 1. The classical Cartan domains (bounded irreducible symmetric domains) are holomorphically homogeneous complete circular domains, and therefore Lu Qi-Keng domains. Thus the representative functions of them are isomorphisms. For instance, the representative function of the domain $R(I)=\left\{z \mid I_{n}-z^{*} z>0, z:(m \times n)\right.$ matrix variable\} (first type of the Cartan domains) under the condition $w\left(z_{0}\right)=0(\tau=0)$ is given by

$$
\widetilde{w}(z)=T_{R(I)}^{-1}\left(z_{0}, \bar{z}_{0}\right) \int_{z_{0}}^{z} T_{R(\bar{l})}\left(z, \bar{z}_{0}\right) d \widetilde{z}, \quad z_{0} \in R(I),
$$

where $z=\left(z_{i j}\right)(i=1,2, \cdots, m ; j=1,2, \cdots, n), \quad \widetilde{z}={ }^{t}\left(z_{11}, z_{12}, \cdots, z_{1 n}\right.$, $\left.z_{21}, \cdots, z_{2 n}, \cdots, z_{m 1}, \cdots, z_{m n}\right)$ and

$$
T_{R(I)}\left(z, \bar{z}_{0}\right)=(m+n)^{t}\left(I_{n}-z_{0}^{*} z\right)^{-1} \times\left(I_{m}-z z_{0}^{*}\right)^{-1} .
$$

By direct calculations we have

$$
w(z)=\left(I_{m}-z_{0} z_{0}^{*}\right)^{-1}\left(z-z_{0}\right)\left(I_{n}-z_{0}^{*} z\right)^{-1}\left(I_{n}-z_{0}^{*} z_{0}\right)
$$

(see [8], p. 13).

Noting that $(A \times B)^{-1}=A^{-1} \times B^{-1},{ }^{t}\left(A^{-1}\right)=\left({ }^{t} A\right)^{-1}$, we have

(19) $d \widetilde{w}(z) / d \widetilde{z}=T_{R(I)}^{-1}\left(z_{0}, \bar{z}_{0}\right) T_{R(I)}\left(z, \bar{z}_{0}\right)$

$$
=\left\{{ }^{t}\left(I_{n}-z_{0}^{*} z_{0}\right) \times\left(I_{m}-z_{0} z_{0}^{*}\right)\right\}\left\{^{t}\left(I_{n}-z_{0}^{*} z\right)^{-1} \times\left(I_{m}-z z_{0}^{*}\right)^{-1}\right\} .
$$

Thus we have $\operatorname{det}(d \widetilde{w}(z) / d \widetilde{z}) \neq 0, \infty$ in $R(I)$, and the representative function (18) is globally one-to-one.

A holomorphic homogeneous transformation $\eta=\eta(z)\left(\eta\left(z_{0}\right)=\tau\right)$ in 
$R(I)$ is given by

$$
\begin{aligned}
& \left(I_{m}-z_{0} z_{0}^{*}\right)^{-1 / 2}\left(z-z_{0}\right)\left(I_{n}-z_{0}^{*} z\right)^{-1}\left(I_{n}-z_{0}^{*} z_{0}\right)^{1 / 2} \\
& \quad=U_{0}\left(I_{m}-\tau \tau^{*}\right)^{-1 / 2}(\eta-\tau)\left(I_{n}-\tau^{*} \eta\right)^{-1}\left(I_{n}-\tau^{*} \tau\right)^{1 / 2} U_{1},
\end{aligned}
$$

where $U_{0}$ and $U_{1}$ are constant unitary matrices, as is well known ([8]). The representative function (18) is very close to the holomorphic homogeneous transformation (20) for $\tau=0$.

The Maschler's m-representative function ([7]) and the $m$-representative function of Kato [4] or Kikuchi [5] of $D$ under the same initial conditions $\left(w\left(z_{0}\right), d w\left(z_{0}\right) / d z, d^{2} w\left(z_{0}\right) / d z^{2}, \cdots, d^{m} w\left(z_{0}\right) / d z^{m}\right)=\left(0, I_{n}\right.$, $0, \cdots, 0), z_{0} \in D$, are given as follows:

$$
w(z)=\left(0, I_{n}, 0, \cdots, 0\right) P_{m}\left(z, \bar{z}_{0}\right) /(1,0, \cdots, 0) P_{m-1}\left(z, \bar{z}_{0}\right),
$$

where

$$
\begin{gathered}
P_{i}\left(z, \bar{z}_{0}\right)=\left[\begin{array}{ccc}
k_{00^{*}} & \cdots & k_{i 0^{*}} \\
\vdots & & \vdots \\
k_{0 i^{*}} & \cdots & k_{i i^{*}}
\end{array}\right]^{-1}\left[\begin{array}{l}
k_{00^{*}}\left(z, \bar{z}_{0}\right) \\
\vdots \\
k_{0 i^{*}}\left(z, \bar{z}_{0}\right)
\end{array}\right], \\
k_{i j^{*}}=\partial^{i+j} k_{D}(z, \bar{t}) /\left.\partial t^{* j} \partial z^{i}\right|_{z=t=z_{0}} \text { and } k_{0 j^{*}}\left(z, \bar{z}_{0}\right)=\partial^{j} k_{D}(z, \bar{t}) /\left.\partial t^{* j}\right|_{t=z_{0}},
\end{gathered}
$$

and

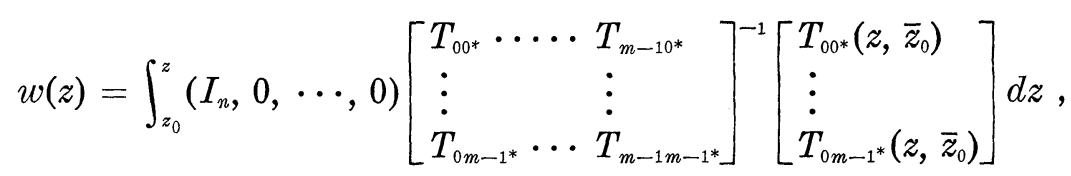

where $T_{i j^{*}}=\partial^{i+j} T_{D}(z, \bar{t}) /\left.\partial t^{* j} \partial z^{i}\right|_{z=t=z_{0}}$ and $T_{0 j^{*}}\left(z, \bar{z}_{0}\right)=\partial^{j} T_{D}(z, \bar{t}) /\left.\partial t^{* j}\right|_{t=z_{0}}$, respectively. Here the $i$ th partial differentiation of a function is defined as in [4], [13]. When $m$ is equal to 1, (21) and (22) coincide with the Bergman representative function.

CoRollary 2. The m-representative function (22) ( $m \geqq 2)$ of a bounded complete circular domain $D$ with center at the origin is holomorphic in $D$ if $\operatorname{det}\left(T_{i j^{*}}\right) \neq 0$ at $z_{0}$.

Proof. By Theorem 1 and (13) $T_{D}(z, \bar{t})$ is holomorphic in $D \times D^{*}$ and $\partial^{j} T_{D}(z, \bar{t}) / \partial t^{* j}(j \geqq 1)$ are so. Therefore, if $\operatorname{det}\left(T_{i j^{*}}\right) \neq 0$, (22) exists and is holomorphic.

EXAMPLE 2. The 2-representative function (22) of a unit dise with respect to $z_{0}(\neq 0) \in D$ is constructed by using $k_{D}\left(z, \bar{z}_{0}\right)=$ $1 / \pi\left(1-\bar{z}_{0} z\right)^{2}$ and $T_{D}\left(z, \bar{z}_{0}\right)=2 /\left(1-\bar{z}_{0} z\right)^{2}$ as follows:

$$
w(z)=\left(1-\left|z_{0}\right|^{2}\right)\left(1-\bar{z}_{0} u\right) u, \quad u=\left(z-z_{0}\right) /\left(1-\bar{z}_{0} z\right) .
$$


The 2-representative function (23) (see [4], [5]) is holomorphic in $D$ with respect to an arbitrary point $z_{0} \in D$, but is not locally one-to-one for any $z_{0}$ in $1 / 2<\left|z_{0}\right|<1$, because at such $z_{0} d w(z) / d z=\left(1-\left|z_{0}\right|^{2}\right)^{2}$ $\left(1+2\left|z_{0}\right|^{2}-3 \bar{z}_{0} z\right) /\left(1-\bar{z}_{0} z\right)^{3}=0$ has a solution $z=\left(1+2\left|z_{0}\right|^{2}\right) / 3 \bar{z}_{0}$ in $|\boldsymbol{z}|<1$.

The Maschler's 2-representative function (21) (for $m=2$ ) of a unit disc with respect to $z_{0} \in D$ is given by

$$
w(z)=\left(1-\left|z_{0}\right|^{2}\right)\left(1-3 \bar{z}_{0} u\right) u /\left(1-2 \bar{z}_{0} u\right), \quad u=\left(z-z_{0}\right) /\left(1-\bar{z}_{0} z\right),
$$

([4]). It is easily seen that (24) is meromorphic for any $z_{0}$ in $1 / 2<$ $\left|z_{0}\right|<1$. And further (24) is not in general locally one-to-one, because $d w(z) / d z=\left(1-\left|z_{0}\right|^{2}\right)^{2}\left(1-6 \bar{z}_{0} u+6 \bar{z}_{0}^{2} u^{2}\right) /\left(1+2\left|z_{0}\right|^{2}-3 \bar{z}_{0} z\right)^{2}=0$ has a solution $z=\left(1+(3+\sqrt{3})\left|z_{0}\right|^{2}\right) /(4+\sqrt{3}) \bar{z}_{0}$ in $|z|<1$ for $z_{0}$ in $1 /(3+\sqrt{3})<$ $\left|z_{0}\right|<1$ and has two solutions $z=\left(1+(3 \pm \sqrt{3})\left|z_{0}\right|^{2}\right) /(4 \pm \sqrt{3}) \bar{z}_{0}$ in $|z|<1$ for $z_{0}$ in $1 /(3-\sqrt{3})<\left|z_{0}\right|<1$. Indeed, from $d w(z) / d z=0$ we have $\bar{z}_{0} u=\left(\bar{z}_{0} z-\left|z_{0}\right|^{2}\right) /\left(1-\bar{z}_{0} z\right)=1 /(3 \pm \sqrt{3})$, and noting that $|z|<1$ we have the result.

\section{REFERENCES}

1. D. Bell, Some properties of the Bergman kernel function, Compositio Math., 21 Fasc. 4 (1969), 329-330.

2. S. Bergman, The kernel function and conformal mapping, Math. Surveys, Amer. Math. Soc., 5, 1950.

3. B. A. Fuks, Theory of analytic functions of several complex variables, Transl. Math. Monographs, Vol. 8, Amer. Math. Soc., 1963.

4. S. Kato, Canonical domains in several complex variables, Pacific J. Math., 21 (1967), 279-291.

5. K. Kikuchi, Various m-representative domains in several complex variables, Pacific J. Math., 33 (1970), 677-692.

6. M. Maschler, Minimal domains and their Bergman kernel function, Pacific J. Math., 6 (1956), 501-516.

7. Classes of minimal and representative domains and their kernel functions, Pacific J. Math., 9 (1959), 763-782.

8. S. Matsuura, On the normal domains and geodesics in the bounded symmetric spaces and the projective space, Sci. Rep. Gunma Univ., 15 (1966), 1-21.

9. - Bergman kernel functions and the three types of canonical domains, Pacific J. Math., 33 (1970), 363-384.

10. Lu Qi-Keng, On Kaehler manifolds with constant curvature, Acta Math. Sinica, 16 (1966), 269-281.

11. P. Rosenthal, On the zeros of the Bergman function in doubly-connected domains, Proc. Amer. Math. Soc., 21 (1969), 33-35.

12. M. Skwarczynski, The invariant distance in the theory of pseudo-conformal transformations and the Lu Qi-Keng conjecture, Proc. Amer. Math. Soc., 22 (1969), 305-310. 13. T. Tsuboi, n-representative domains and $n$-minimal domains in several complex variables, Sci. Rep. Saitama Univ., A 4 (1961), 1-14.

14. T. Tsuboi and S. Matsuura, Some canonical domains in $C^{n}$ and moment of inertia theorems, Duke Math. J., 36 (1969), 517-536.

Received December 23, 1971.

Nagoya Institute of TEChNOLOGY

NAGOYA, JAPAN 


\section{PACIFIC JOURNAL OF MATHEMATICS}

\section{EDITORS}

RICHARD ARENS (Managing Editor)

University of California

Los Angeles, California 90024

\section{R. A. Beaumont}

University of Washington

Seattle, Washington 98105

\section{J. DuGundJI*}

Department of Mathematics

University of Southern California

Los Angeles, California 90007

D. Gilbarg and J. Milgram

Stanford University

Stanford, California 94305

ASSOCIATE EDITORS
E. F. BECKENBACH
B. H. NeumanN
F. WOLF
K. YoSHIDA

\section{SUPPORTING INSTITUTIONS}

UNIVERSITY OF BRITISH COLUMBIA

CALIFORNIA INSTITUTE OF TECHNOLOGY

UNIVERSITY OF CALIFORNIA

MONTANA STATE UNIVERSITY

UNIVERSITY OF NEVADA

NEW MEXICO STATE UNIVERSITY

OREGON STATE UNIVERSITY

UNIVERSITY OF OREGON

OSAKA UNIVERSITY

\author{
UNIVERSITY OF SOUTHERN CALIFORNIA \\ STANFORD UNIVERSITY \\ UNIVERSITY OF TOKYO \\ UNIVERSITY OF UTAH \\ WASHINGTON STATE UNIVERSITY \\ UNIVERSITY OF WASHINGTON \\ AMERICAN MATHEMATICAL SOCIETY \\ NAVAL WEAPONS CENTER
}

The Supporting Institutions listed above contribute to the cost of publication of this Journal, but they are not owners or publishers and have no responsibility for its content or policies.

Mathematical papers intended for publication in the Pacific Journal of Mathematics should be in typed form or offset-reproduced, (not dittoed), double spaced with large margins. Underline Greek letters in red, German in green, and script in blue. The first paragraph or two must be capable of being used separately as a synopsis of the entire paper. Items of the bibliography should not be cited there unless absolutely necessary, in which case they must be identified by author and Journal, rather than by item number. Manuscripts, in duplicate if possible, may be sent to any one of the four editors. Please classify according to the scheme of Math. Rev. Index to Vol. 39. All other communications to the editors should be addressed to the managing editor, or Elaine Barth, University of California, Los Angeles, California, 90024.

50 reprints are provided free for each article; additional copies may be obtained at cost in multiples of 50 .

The Pacific Journal of Mathematics is issued monthly as of January 1966. Regular subscription rate: $\$ 48.00$ a year (6 Vols., 12 issues). Special rate: $\$ 24.00$ a year to individual members of supporting institutions.

Subscriptions, orders for back numbers, and changes of address should be sent to Pacific Journal of Mathematics, 103 Highland Boulevard, Berkeley, California, 94708.

\section{PUBLISHED BY PACIFIC JOURNAL OF MATHEMATICS, A NON-PROFIT CORPORATION}

Printed at Kokusai Bunken Insatsusha (International Academic Printing Co., Ltd.), 270, 3-chome Totsuka-cho, Shinjuku-ku, Tokyo 160, Japan.

* C. R. DePrima California Institute of Technology, Pasadena, CA 91109, will replace J. Dugundji until August 1974.

Copyright (C) 1973 by

Pacific Journal of Mathematics

All Rights Reserved 


\section{Pacific Journal of Mathematics}

\section{Vol. 49, No. $2 \quad$ June, 1973}

Wm. R. Allaway, On finding the distribution function for an orthogonal polynomial

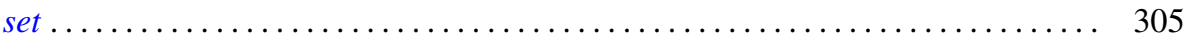

Eric Amar, Sur un théorème de Mooney relatif aux fonctions analytiques bornées... . 311

Robert Morgan Brooks, Analytic structure in the spectrum of a natural system . . . . 315

Bahattin Cengiz, On extremely regular function spaces . . . . . . . . . . . . . . 335

Kwang-nan Chow and Moses Glasner, Atoms on the Royden boundary . . . . . . . . . 339

Paul Frazier Duvall, Jr. and Jim Maxwell, Tame $Z^{2}$-actions on $E^{n} \ldots \ldots \ldots \ldots \ldots . .349$

Allen Roy Freedman, On the additivity theorem for $n$-dimensional asymptotic

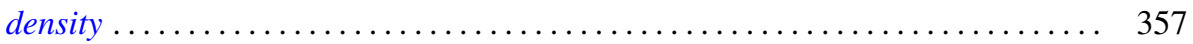

John Griffin and Kelly Denis McKennon, Multipliers and the group $L_{p}$-algebras . . . 365

Charles Lemuel Hagopian, Characterizations of $\lambda$ connected plane continua ....... 371

Jon Craig Helton, Bounds for products of interval functions . . . . . . . . . . . 377

Ikuko Kayashima, On relations between Nörlund and Riesz means . . . . . . . . . . 391

Everett Lee Lady, Slender rings and modules . . . . . . . . . . . . . . . . . . 397

Shozo Matsuura, On the Lu Qi-Keng conjecture and the Bergman representative

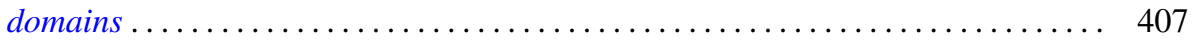

Stephen H. McCleary, The lattice-ordered group of automorphisms of an $\alpha$-set . . . 417

Stephen H. McCleary, o-2-transitive ordered permutation groups .......... 425

Stephen H. McCleary, o-primitive ordered permutation groups. II . . . . . . . . . 431

Richard Rochberg, Almost isometries of Banach spaces and moduli of planar

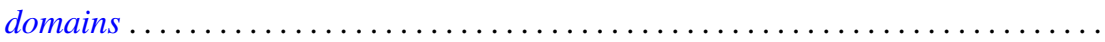

R. F. Rossa, Radical properties involving one-sided ideals . . . . . . . . . . . . . 467

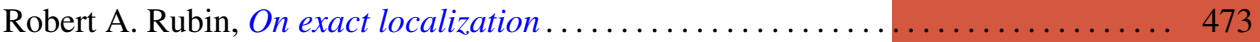

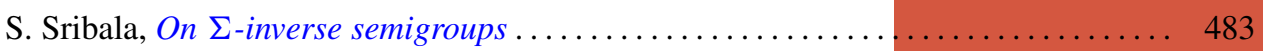

H. M. (Hari Mohan) Srivastava, On the Konhauser sets of biorthogonal polynomials suggested by the Laguerre polynomials ...................... 489

Stuart A. Steinberg, Rings of quotients of rings without nilpotent elements . ...... 493

Daniel Mullane Sunday, The self-equivalences of an $H$-space . . ............ 507

W. J. Thron and Richard Hawks Warren, On the lattice of proximities of $\check{C} e c h$

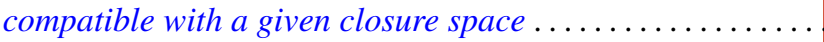

Frank Uhlig, The number of vectors jointly annihilated by two real quadratic forms determines the inertia of matrices in the associated pencil .

Frank Uhlig, On the maximal number of linearly independent real vectors annihilated simultaneously by two real quadratic forms ..............

Frank Uhlig, Definite and semidefinite matrices in a real symmetric matrix pencil . . 561

Arnold Lewis Villone, Self-adjoint extensions of symmetric differential operators . . . 569

Cary Webb, Tensor and direct products . ....................... 579

James Victor Whittaker, On normal subgroups of differentiable

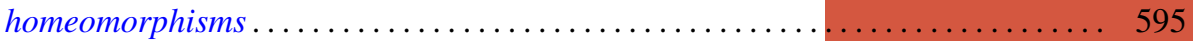

Jerome L. Paul, Addendum to: "Sequences of homeomorphisms which converge to

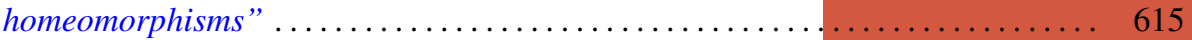

David E. Fields, Correction to: “Dimension theory in power series rings” ........ 616

Peter Michael Curran, Correction to: “Cohomology of finitely presented groups”. . . 617

Billy E. Rhoades, Correction to: “Commutants of some Hausdorff matrices” ...... 617

Charles W. Trigg, Corrections to: "Versum sequences in the binary system” ...... 619 